Pathologe $2021 \cdot 42: 164-171$

https://doi.org/10.1007/s00292-021-00918-9

Angenommen: 13. Januar 2021

Online publiziert: 9. Februar 2021

(c) Springer Medizin Verlag GmbH, ein Teil von

Springer Nature 2021

\section{Schwerpunktherausgeber}

W. Roth, Mainz

P. Boor, Aachen

\author{
Maximilian Ackermann ${ }^{1,2} \cdot$ Christopher Werlein ${ }^{3,4} \cdot$ Florian Länger $^{3,4}$. \\ Mark P. Kühnel ${ }^{3,4}$ - Danny D. Jonigk ${ }^{3,4}$ \\ 'Institut für Pathologie und Molekularpathologie, Helios Universitätsklinikum Wuppertal, Universität \\ Witten-Herdecke, Wuppertal, Deutschland \\ ${ }^{2}$ Institut für Funktionelle und Klinische Anatomie, Universitätsmedizin der Johannes Gutenberg- \\ Universität Mainz, Mainz, Deutschland \\ ${ }^{3}$ Institut für Pathologie, Medizinische Hochschule Hannover (MHH), Hannover, Deutschland \\ ${ }^{4}$ Deutsches Zentrum für Lungenforschung (DZL), Biomedical Research in Endstage and Obstructive Lung \\ Disease (BREATH), Hannover, Deutschland
}

\title{
COVID-19: Auswirkungen auf Lunge und Herz
}

flächenantigenstrukturen, welche aber trotzdem mutmaßlich allen großen Pandemiewellen vorausgehen, v. a. wenn es $\mathrm{zu}$ einem Genrearrangement zwischen humanen und tierpathogenen Viren kommt.

Viele Studien legen nahe, dass auch ein solcher evolutionärer , antigen shift" Ursprung des von der Weltgesundheitsorganisation WHO als globale Pandemie eingestuften SARS-CoV-2-Ausbruchs anzusehen ist [2]. Nach Beschreibung von schweren, zunächst unklaren Pneumonien in Wuhan in China im Dezember 2019 wurde nach Sequenzierung das SARSCoV-2-Virus phylogenetisch der Familie der Coronaviren zugeordnet. Bei den Coronaviridae handelt es sich um Einzelstrang-RNA-Viren mit einem Durchmesser von $80-120 \mathrm{~nm}$, welche schon für die SARS-CoV-1-Epidemie 2003 und den epidemischen Ausbruch von MERS$\mathrm{CoV}$ im Jahr 2013 verantwortlich waren. Das aktuelle SARS-CoV-2-Virus weistallerdings im Vergleich zu SARS-CoV-1 und MERS-CoV eine höhere Reproduktionsrate bei gleichzeitig geringerer Mortalität auf. Wie bei nahezu allen Coronaviren besteht ein ausgeprägter Tropismus für den $\mathrm{ACE}$ (Angiotensin-konvertierendes Enzym)-Rezeptor 2, der neben dem Epithel des oberen und unteren Respirationstraktes unter anderem auch auf Endothelien und den Epithelien viszeraler Organe zu finden ist. Obgleich bei schweren Verläufen die SARS-CoV-2-Pneumonie zu einem akuten Lungenversagen führen kann, zeigen sich bei vielen Patienten in unterschiedlicher Häufigkeit auch andere Organmanifestationen in Herz, Niere, Gehirn und Leber, sodass man bei COVID-19 von einer vaskulären Multiorganerkrankung sprechen kann $[3,4]$.

COVID-19 wird häufig mit einer schweren Influenza verglichen und bei bis zu $20 \%$ der Patienten mit SARSCoV-2-Infektion werden schwere Verläufe beobachtet, die eine intensivmedizinische Versorgung im Rahmen eines "acute respiratory distress syndrome" (ARDS, akutes Lungenversagen) erforderlich machen [5]. In schweren Fällen kommt es bei beiden Erkrankungen zu einem akuten Lungenversagen, welches mit einer Störung des pulmonalen Gasaustausches einhergeht und häufig eine mechanische Beatmung erforderlich macht. Allerdings zeigt COVID19 interessanterweise keine klinischen Merkmale eines "typischen" ARDS, wie es anästhesiologisch gemäß der BerlinKriterien (u.a. verminderte Lungencompliance, Horovitz-Quotient aus arteriellem Sauerstoff-Partialdruck $\left[\mathrm{P}_{\mathrm{a}} \mathrm{O}_{2}\right]$ und der inspiratorischen Sauerstofffraktion $\left[\mathrm{F}_{\mathrm{i}} \mathrm{O}_{2}\right]$, bilaterale Verdichtungen im CT) klassifiziert wurde, da viele Patienten trotz signifikanter Hypoxämie keine Dyspnoesymptomatik aufweisen und mechanische Compliancewerte im Normbereich zeigen. Es wird vermutet, dass hier die respiratorische Insuffizienz über eine Umverteilung der Ventilation und Perfusion kompensiert wird. Pathophysiologisch kann dies durch den Euler-Liljestrand-Mechanismus erklärt 

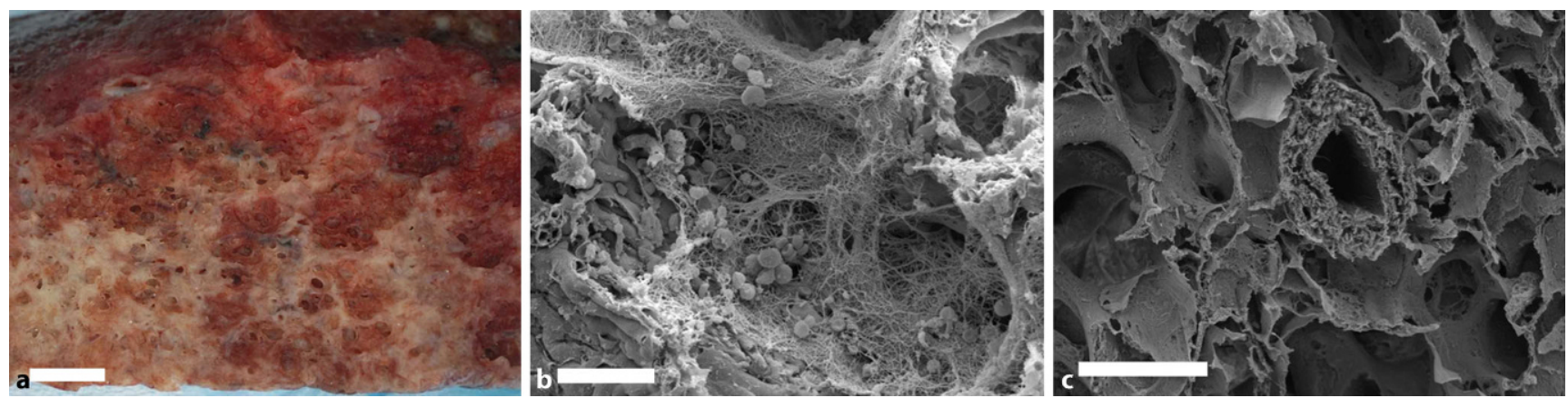

Abb. $1 \Delta$ a Makroskopisch erkennt man bei COVID-19-Autopsien stark vaskularisierte, ödematöse Lungen im frühen Akutstadium (Tag 7 nach Infektion). Rasterelektronenmikroskopische Aufnahmen zeigen, dass das parenchymatöse Grundgerüst bei COVID-19-Lungen (b) ähnlich wie bei normalen Lungengewebe (c) weitgehend erhalten ist. Man findet jedoch einen beginnenden Alveolarschaden mit Fibrinexsudaten und Hämorrhagien. Balken a: 1 cm, b: $20 \mu \mathrm{m}, \mathrm{c:}: 200 \mu \mathrm{m}$

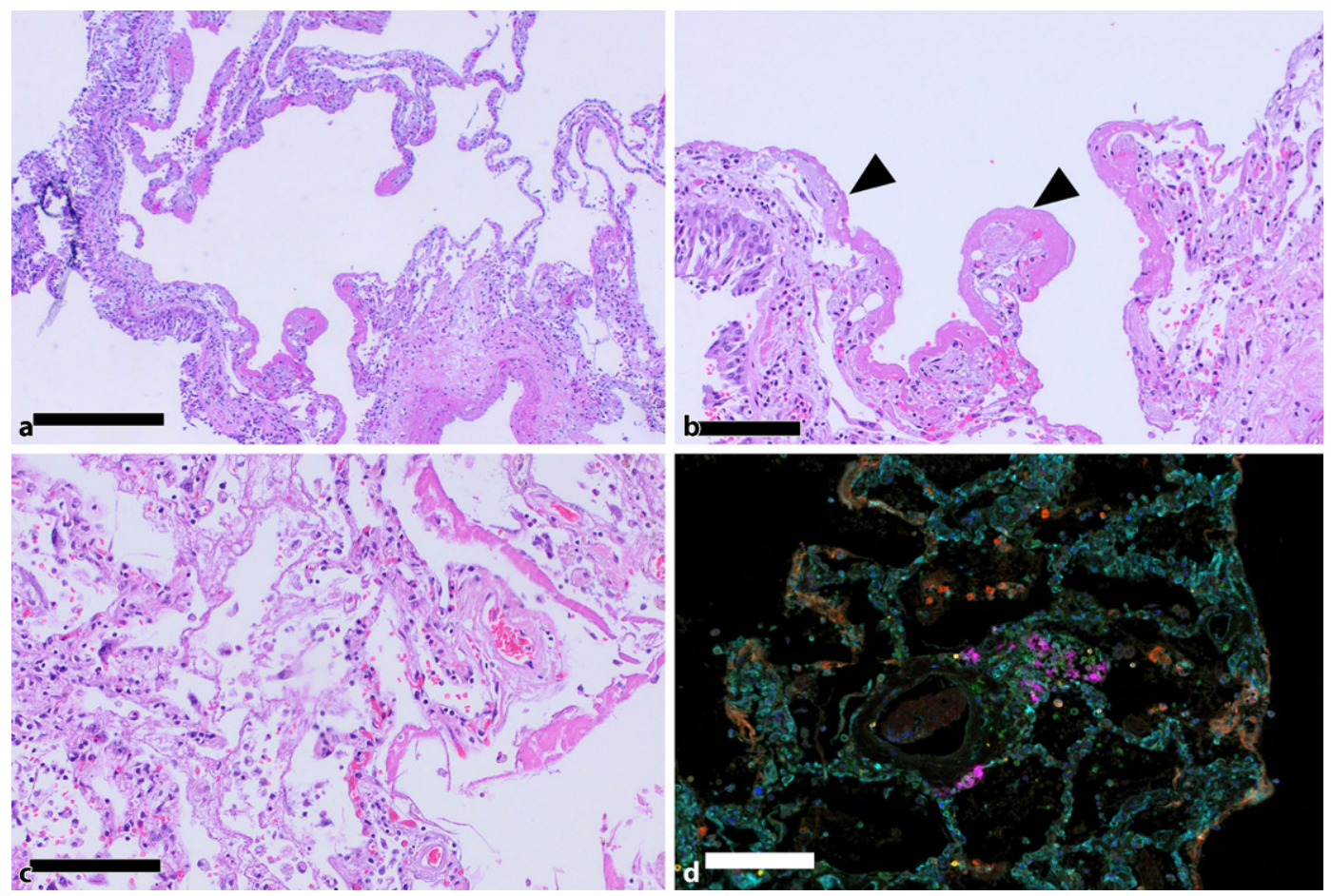

Abb. 2 \ Diffuser Alveolarschaden bei COVID-19- und Influenzapneumonie. a, b Bei der COVID-19-Pneumonie (Tag 9 nach Infektion) findet man vorwiegend flächenhafte hyaline Membranen (Pfeilspitzen) ein interstitielles Lungenödem und eine interstitielle Pneumonitis. Herdförmig lässt sich auch bereits ein beginnender fibrotischer Parenchymumbau mit Akkumulation von aktivierten Myofibroblasten erkennen. c Lungengewebe von Influenzapatienten (Tag 10 nach Infektion) stellt sich meist mit ähnlichen Veränderungen dar, wobei die Gewebedestruktion durch eine nahezu vollständige Aufhebung der Gewebestruktur mit intraalveolären Fibrinexsudationen und Einblutungen einhergehen. d Multi-Immunfluoreszenzaufnahmen des entzündlichen Infiltrates bei einer COVID-19-Pneumonie (Tag 8 nach Infektion). Es zeigt sich ein ausgeprägtes CD4-positives perivaskuläres Infiltrat. Gelb $=\mathrm{CD} 8$-positive Zellen, grün $=\mathrm{CD} 4$-positive Zellen, pink $=\mathrm{CD} 20$-positive Zellen, orange $=\mathrm{CD68}$ positive Alveolarmakrophagen, blau $=$ neutrophile Granulozyten. Balken $\mathbf{a}=400 \mu \mathrm{m}, \mathbf{b}-\mathbf{d}=200 \mu \mathrm{m}$

werden, bei dem es wechselseitig zu einer reflektorischen Vasokonstriktion der pulmonalarteriellen Strombahn und Hypoventilation der Lungenläppchen kommt.

\section{COVID-19-Pneumonie}

Der Verlauf eines klassischen ARDS folgt meist 3 Phasen:

1. exsudative Phase (Tag 1-3 post infectionem),

2. proliferative Phase (bis zu einer Woche nach Infektion),
3. fibrotisches Remodeling ( $a b$ Tag 7 bis 10 post infectionem).

Auch wenn die COVID-19-Pneumonie in ihren initialen Phasen Ähnlichkeiten zu anderen viralen Atemwegserkrankungen aufweist, sind jedoch im weiteren Verlauf Unterschiede zu beobachten. 
In der Akutphase (bis zum 10. Tag post infectionem) kommt es $\mathrm{zu}$ einer serofibrinösen Entzündungsreaktion in Form einer exsudativen Alveolitis durch progrediente Schädigung der Pneumozyten und Kapillarendothelien [3]. Durch die Destruktion der alveolokapillären Membran, die Freisetzung von Entzündungsmediatoren im Rahmen des Zytokinsturms und eine erhöhte vaskuläre Permeabilität wird ein überwiegend interstitielles, teils im schwerem Verlauf auch intraalveoläres Ödem ausgelöst, welches die autoptisch durchweg erhöhten postmortalen Lungengewichte von COVID-19 von kombiniert meist über $2 \mathrm{~kg}$ erklärt (• Abb. 1; [3, 6, 7]). Das histopathologische Korrelat des klinisch meist als todesursächlich beschriebenen akuten Lungenversagens stellt ein unspezifischer diffuser Alveolarschaden dar, der insbesondere durch hyaline Membranen, interstitielle Ödembildung, Einblutungen und intraalveoläre Fibrinablagerungen ( Abb. 1) charakterisiert ist. In der Spätphase kommt es bei der anhaltenden Schädigung zu Mikro- und Makroatelektasen, einer Hyperplasie der Typ-2-Pneumozyten sowie im Interstitium herdförmig zur Ansammlung von Myofibroblasten (• Abb. 2). Diese morphologischen Schädigungsmuster sind allerdings nicht pathognomonisch für die COVID-19-Pneumonie, sondern werden auch bei anderen Lungenerkrankungen, wie z. B. bei der Influenzapneumonie, vorgefunden [3].

Neben der komplexen Schädigung des Alveolarepithels hebt sich die COVID19-Pneumonie insbesondere durch die ausgeprägte angiozentrische Inflammation von anderen Pneumonien $a b$ (- Abb. 2). SARS-CoV-2 kann über virale Spikeproteine (Spike Glykoprotein S1 der Rezeptorbindungsdomäne) an die Bindungsstelle des Rezeptors von ACE2 sowie an den Korezeptor Neuropilin-1 binden, welches in der Lunge neben Alveolar- und Bronchusepithel und Makrophagen insbesondere auf Endothelzellen und Perizyten exprimiert wird (- Abb. 3; [3]). Nach Eintritt von SARSCoV-2 in die Wirtszelle kommt es in kürzester Zeit zu einer viralen Replikation im endoplasmatischen Retikulum, der Assemblierung mit viralen Struk-

Pathologe 2021 - 42:164-171 https://doi.org/10.1007/s00292-021-00918-9

(c) Springer Medizin Verlag GmbH, ein Teil von Springer Nature 2021

\section{Ackermann · C. Werlein · F. Länger · M. P. Kühnel • D. D. Jonigk}

\section{COVID-19: Auswirkungen auf Lunge und Herz}

\section{Zusammenfassung}

Neben der jährlich saisonal auftretenden Influenzaendemie stellt das sich pandemisch verbreitende SARS-CoV-2-Virus die weltweiten Gesundheitssysteme als vaskuläre Multiorganerkrankung COVID-19 vor ganz neue medizinische und sozioökonomische Herausforderungen. Bei beiden Viruserkrankungen kommt es insbesondere bei schweren Krankheitsverläufen zu einem fulminanten Lungenversagen, dem "acute respiratory distress syndrome" (ARDS), welchem morphologisch meist ein unspezifischer diffuser Alveolarschaden mit hyalinen Membranen, Fibrinexsudaten und einem intraalveolären Ödem zugrunde liegt. Neben einer Infektion des Alveolarepithels und von Makrophagen durch das SARS-CoV-2-Virus scheint die COVID-19-Erkrankung zusätzlich durch eine vaskulozentrische Entzündungsreaktion mit Mikroangiopathie und aberranter Blutgefäßneubildung durch die sog. intussuszeptive Angiogenese charakterisiert zu sein. Zudem finden sich wahrscheinlich auf dem Boden dieser primär systemischen angiozentrischen Entzündung in vielen weiteren Organen, z. B. dem Herzen, im unterschiedlichen Ausmaß Gewebeschädigungen.

Schlüsselwörter

Humane Influenza · Pneumonie · Lungenversagen $\cdot$ SARS-CoV-2 $\cdot$ Angiogenese

\section{COVID-19: effects on the lungs and heart}

\section{Abstract}

Viral respiratory diseases constitute the most common reasons for hospitalization with more than half of all acute illnesses worldwide. Progressive respiratory failure with pronounced diffuse alveolar damage has been identified as the primary cause of death in COVID-19. COVID-19 pneumonia shares common histopathological hallmarks with influenza (H1N1)-related ARDS, like diffuse alveolar damage (DAD) with edema, hemorrhage, and intra-alveolar fibrin deposition. The lungs with COVID-19 pneumonia revealed perivascular inflammation, an endothelial injury, microangiopathy, and an aberrant blood vessel neoformation by intussusceptive angiogenesis. While this pronounced angiocentric inflammation is likely be found - to varying degrees in numerous other organs, e.g., the heart, COVID-19 is hypothesized to be not just a pulmonary, but rather a systemic "vascular disease."

\section{Keywords}

Human influenza Pneumonia $\cdot$ Respiratory distress syndrome - SARS-CoV-2 . Angiogenesis turproteinen und der Exozytose aus der Wirtszelle, was eine ausgeprägte Virämie ab ca. dem 5. Tag nach Infektion zur Folge hat. Neben einer direkten apoptotischen Schädigung durch die Replikation von SARS-CoV-2 kommt es ebenfalls zu einer adaptiven und T-Zellvermittelten Immunreaktion. In eigenen morphometrischen Untersuchungen [3] konnten wir mittels Multiplex-Immunofluoreszenzanalyse eine annähernd gleich dichte Infiltration CD3-positiver T-Zellen im Interstitium von Lungengewebe COVID-19- oder Influenza-Verstorbener feststellen (• Abb. 2). Während jedoch in den Influenzaproben vermehrt zytotoxische, CD8-positive T-Lympho- zyten und CD15-positive, neutrophile Granulozyten nachweisbar waren, zeigte sich in der COVID-19-Pneumonie vorwiegend eine perivaskuläre Akkumulation von CD4-positiven T-Helferzellen (• Abb. 2). Die ausgeprägte perivaskuläre T-Zell-vermittelte Entzündung bzw. Endothelialitis korreliert mit der klinischerseits häufig beschriebenen Leukopenie, die sich vorwiegend bei schweren Krankheitsverläufen manifestiert [8]. Die Wechselwirkung zwischen aktivierten T-Zellen und Endothelzellen scheint zum prolongierten interstitiellen Entzündungsverlauf bei COVID-19 beizutragen [9], da aktivierte T-Zellen eine starke Adhärenz an Endothelzellen 


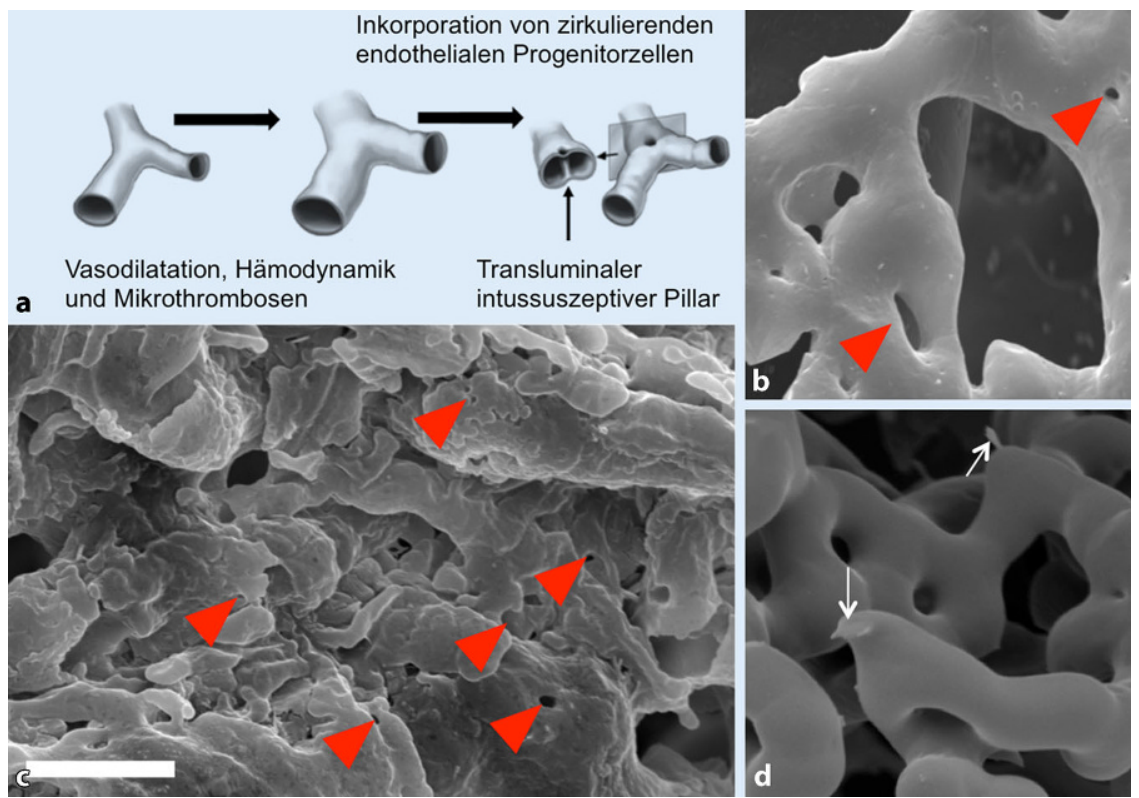

Abb. $3 \Delta$ Intussuszeptive Angiogenese. Die Neubildung kann auf verschiedene Arten erfolgen. Aufgrund von hämodynamischen Veränderungen (z. B. Hypoxie, Inflammation) kommt es zur Rekrutierung von zirkulierenden endothelialen Progenitorzellen, welche in kürzester Zeit dynamisch die Gefäßarchitektur anpassen und optimieren (a). Das morphologische Korrelat dieser intraluminalen Septierung wird in Gefäßausgusspräparaten als „intussuszeptiver Pillar“ (rote Pfeilspitzen) sichtbar. c Bei COVID-19 kommt es zu aberranten Reparaturmechanismen durch eine gesteigerte intussuszeptive Angiogenese (rote Pfeilspitzen, rasterelektronen-mikroskopische Aufnahme eines Gefäßausgusses einer COVID-19-Lunge, Balken: $20 \mu \mathrm{m}$ ). $\mathbf{d}$ Im Gegensatz zur intussuszeptiven Angiogenese kommt es bei der weitaus langsameren sprossenden Angiogenese (weißePfeile) zur Endothelproliferation aus einem existenten Gefäßbaum. (Teilabb. a modifiziert aus Ackkermann et al. [22]. Mit freundlicher Genehmigung European Respiratory Society)

aufweisen, welche vorwiegend über die Sekretion von chemotaktischen Chemokinen vermittelt wird. Darüber hinaus exprimieren entzündlich aktivierte Endothelzellen vermehrt Toll-like-Rezeptoren und MYD88 als wichtige Regulatoren des angeborenen Immunsystems. Diese setzen bei Aktivierung vermehrt proinflammatorische Zytokine (wie z.B. Interferone, TNF- $\alpha$ ), Gefäßzelladhäsionsmoleküle (z.B. E-Selektin, ICAM1, VCAM), prokoagulante Mediatoren (wie z. B. Fibrin, Von-Willebrand-Faktor) und proangiogene Wachstumsfaktoren frei
[10]. SARS-CoV-2-assoziierte Endothelschädigungen und Endothelialitis verursachen in Konsequenz der Schädigung eine überschießende Thrombinund Fibrinfreisetzung, was die in der Pandemie klinisch sehr früh beschriebenen Thrombembolien in mittelgroßen Gefäßen (• Abb.4) und im Kapillarendstromgebiet (๑Abb.4) erklärbar macht. Insgesamt fanden wir 9-mal mehr Mikrothromben in COVID-19-Lungen als bei H1N1-Pneumonien [3]. Neben dieser thrombotischen Mikroangiopathie kann sich in schweren Verläufen zusätzlich noch eine disseminierte intravasale Gerinnung (DIC) entwickeln und es können Immunthromben auftreten [11]. Hierbei kommt es zur Rekrutierung von neutrophilen Granulozyten, die über eine Aktivierung von reaktiven Sauerstoffspezies (ROS) eine Vielzahl ihrer Enzyme, Chromatin sowie knäuelförmige DNA freisetzen. Diese vor einem Jahrzehnt entdeckte Immunantwort der "neutrophil extracellular traps" (NET) bildet somit eine Art ,immunologisches Fangnetz" für Bakterien, Viren und Parasiten, welche über die freigelegten Enzyme enzymatisch degradiert werden.

\section{Endotheliale Dysfunktion und Angiogenese}

Das komplexe Blutgefäßsystem des Körpers, welches sich in seiner Länge über eine Gesamtdistanz von weit mehr als $100.000 \mathrm{~km}$ erstreckt, stellt nicht nur ein elementares Versorgungsnetzwerk des Körpers dar, sondern bildet mit einem Gesamtgewicht von mehr als $2 \mathrm{~kg}$ meist vasoaktiver Endothelzellen eines der größten stoffwechselaktiven Gewebe des Organismus [9]. Im Verlauf der COVID19-Pandemie wurden klinisch sehr früh neben den pulmonalen Manifestationen auch weitere Gefäßbeteiligungen mit anderen Endorganschädigungen, wie z. B. akutes Nierenversagen, beschrieben [12]. Die SARS-CoV-2-assoziierte Funktionsstörung der Endothelzellen scheint bei der Pathogenese dieser schweren klinischen Manifestationen in COVID-19 [3, $9,12]$ von zentraler Bedeutung zu sein. Der zelluläre Eintritt von SARS-CoV-2 wird wie bei anderen SARS-Viren zum großen Teil über den ACE2-Rezeptor vermittelt, der auch von Endothelzellen

Hier steht eine Anzeige. 

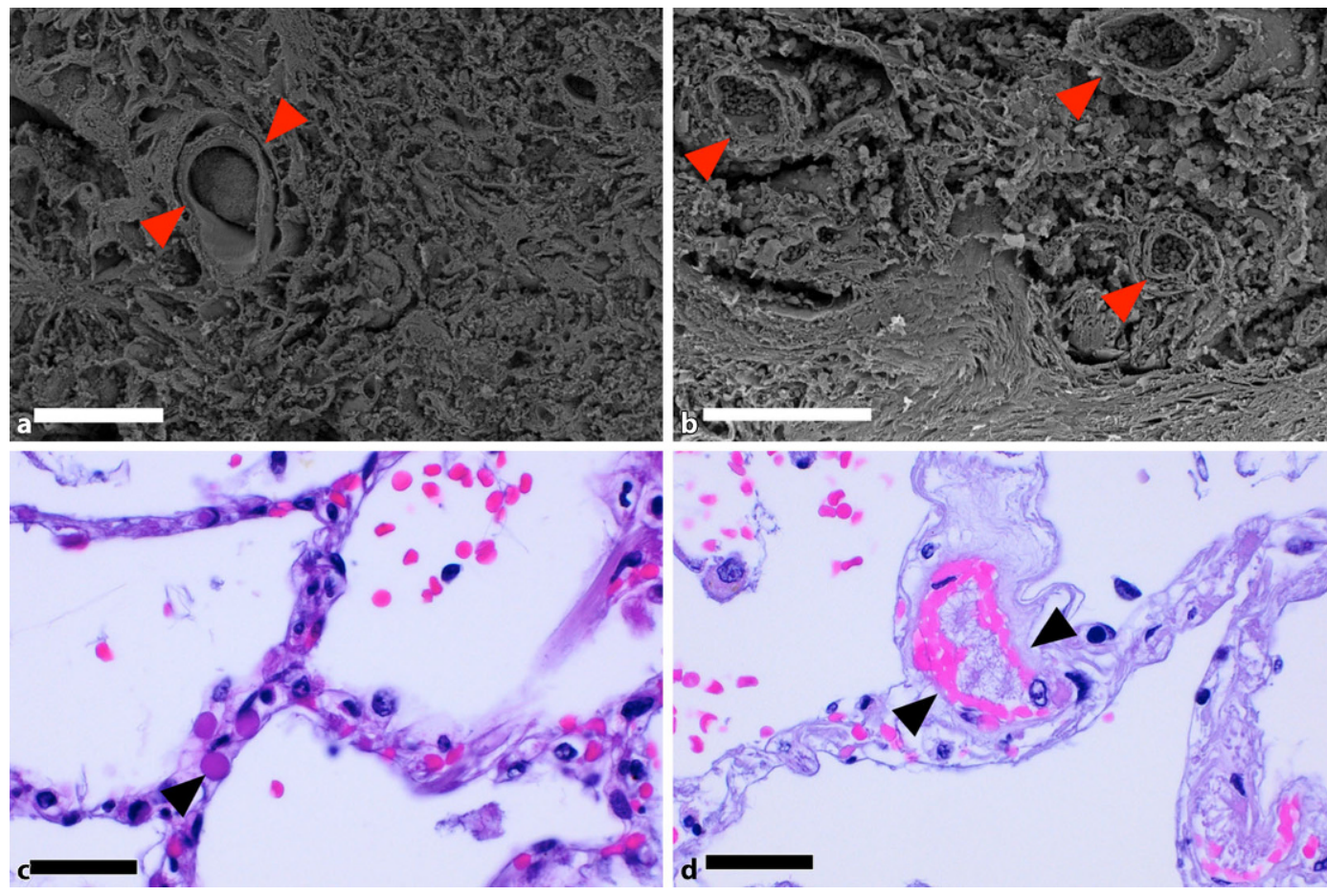

Abb. $4 \varangle$ Rasterelektronenmikroskopische Aufnahmen verdeutlichen die Mikroangiopathie bei COVID-19 mit zahlreichen Mikrothromben in mittelgroßen Venen (rote Pfeilspitzen, a, b). Mittels $\mathrm{HE}$-Färbungen lassen sich zahlreiche venookklusive Thromben (schwarze Pfeilspitzen) innerhalb des kapillären Gefäßplexus (c) ebenso wie in postkapillären Venen darstellen. Balken: a: $500 \mu \mathrm{m}$, b: $100 \mu \mathrm{m} . \mathrm{c}, \mathrm{d}: 20 \mu \mathrm{m}$

in Lunge und Niere exprimiert wird [12]. Die in praktisch allen Organen vorkommende Expression von ACE2 auf Endothelzellen, glatten Muskelzellen und perivaskulären Perizyten legt nahe, dass sich SARS-CoV-2, sobald es im Kreislauf vorhanden ist, leicht im Körper ausbreiten kann [13]. Für einen schweren Verlauf einer COVID-19Pneumonie werden zumeist als Risikofaktoren das Vorliegen einer Arteriosklerose oder Diabetes mellitus diskutiert, denen beide jeweils eine Mikro- und Makroangiopathie mit vorgeschädigtem Endothel zugrunde liegt. Nach Infektion der Alveolarepithelzellen und der Endothelzellen kommt es zur intrazellulären viralen Replikation im endoplasmatischen Retikulum [15-17]. Gleichermaßen unterliegen Zellbestandteile, wie z.B. Zellmembran, Golgi-Apparat oder Mitochondrien, sowie das Zytoskelett virolytischen Veränderungen und Umbauvorgängen [15, 16]. Nach mehrfacher Replikation und Ausschleusung der Viruspartikel aus der Zelle [17] kommt es zur Apoptose $[15,16]$. Die apoptotische Endothelzelle [14, 15] löst sich durch Aufklaffen der engen Zellkontakten, wie z.B. über „tight junctions“, Desmosomen und Hemidesmosomen, von den Nachbarzellen und der Basalmem- bran. Zusätzlich führt die Freisetzung von Zytokinen zum Vascular-Leak-Syndrom, bei dem es durch die erhöhte Permeabilität $\mathrm{zu}$ einem interstitiellen bzw. intraalveolären Ödem kommt.

Das ubiquitär geschädigte Endothel unterliegt einem Reparaturmechanismus, der vorwiegend über die Angiogenese bewerkstelligt wird. Die Blutgefäßneubildung kann grundsätzlich auf 2 Arten erfolgen: Bei der sprossenden Angiogenese („sprouting angiogenesis“) kommt zur Proliferation von Endothelzellen aus einem existenten Kapillarbett entlang eines Wachstumsfaktorgradienten (z.B. VEGF oder FGF). Dieser morphogenetische Prozess nimmt teils mehrere Tage in Anspruch. Bei der intussuszeptiven Angiogenese („intussusceptive angiogenesis“; - Abb. 3) erfolgt hingegen der Umbau und die Expansion des Gefäßbaumes im Gegensatz zur sprossenden Angiogenese ohne Proliferation von Endothelzellen, sondern durch Rekrutierung und Inkorporation von zirkulierenden Progenitorzellen [18]. Die Expansion des Gefäßsystems mittels Intussuszeption tritt dabei überwiegend im venösen Gefäßschenkel oder im Kapillarbett auf. Das morphologische Korrelat dieser endothelialen Invagination bilden transluminale $\mathrm{Ge}$ - webesäulen („pillars“), die in den entsprechenden Gefäßausgusspräparaten in der Rasterelektronenmikroskopie als kleine Löcher mit einem Durchmesser von 1-2 $\mu \mathrm{m}$ imponieren (• Abb. 3). Durch eine Endothelprotrusion entsteht eine intraluminale Gewebefalte, die sich über Myofibroblasten und Perizyten lumenwärts einstülpt, bis sich die beiden gegenüberliegenden Gewebeschichten berühren. Es kommt zur Verschmelzung und Fusion der beiden Membranen der Endothelzellen, bis dies in eine komplette Septierung der neu entstandenen Gefäße mündet. Gleichermaßen werden zirkulierende endotheliale Progenitorzellen bei der Integration in die Gefäßwand beschrieben [18]. Diese zirkulierenden Vorläuferzellen exprimieren unter anderem Merkmale von endothelialen Antigenen, wie z.B. $\mathrm{CD}_{34}, \mathrm{CD}_{133}$ und VEGFR-2, und weisen phänotypische Ähnlichkeiten zu ausgereiften Endothelzellen auf.

Die intussuszeptive Angiogenese tritt bei normalen Entwicklungsprozessen sowie bei vielen pathologischen Zuständen wie Geweberegeneration [19], Entzündung bei Kolitis [20], Myokarditis [21], interstitiellen Lungenerkrankungen [22] und Tumoren [23] auf. Die von uns im Gefäßsystem von COVID-19 beschrie- 

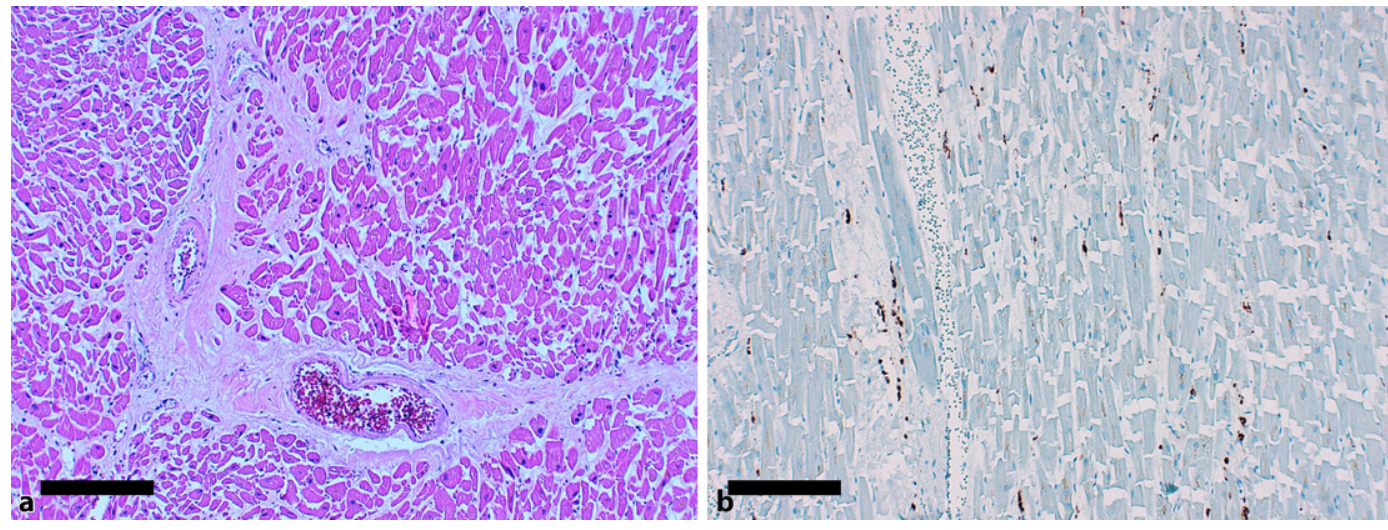

Abb. $5<$ Herzen von COVID-19-Patienten zeigen meist morphologische Zeichen einer unspezifischen Vorschädigung, wie z. B. eine perivaskulär akzentuierte Fibrose (a). Interessanterweise wurde häufig eine diffuse Infiltration von Makrophagen beobachtet (b, anti-CD68 PGM1-Färbung). Balken a, b: $200 \mu \mathrm{m}$

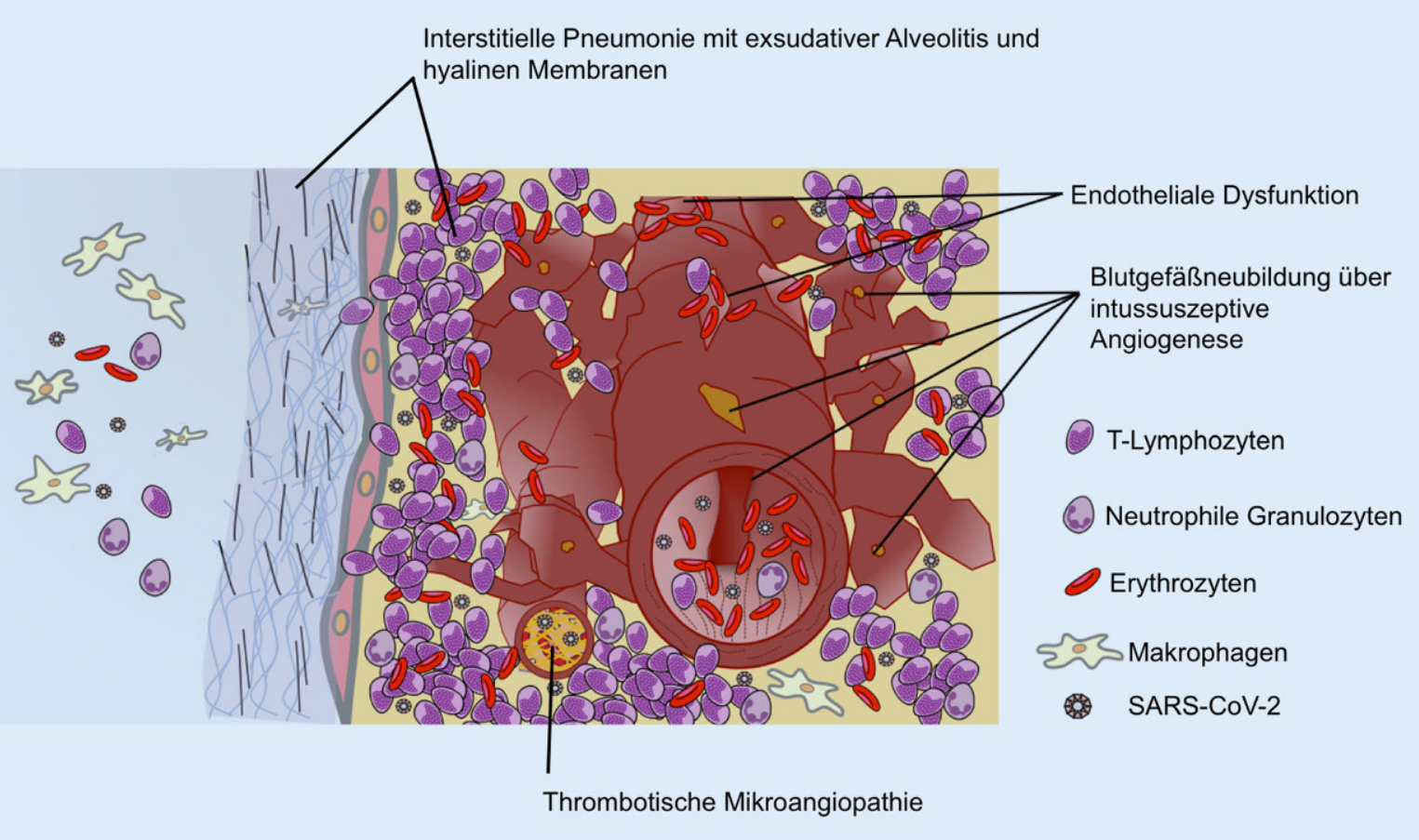

Abb. 6 \ Synopse derCOVID-19-Pneumonie. (Modifiziert nach Ackermann et al. [9]. Mitfreundlicher Genehmigung European Respiratory Society)

bene Neoangiogenese mittels Intussuszeption scheint somit ein aberranter Reparaturmechanismus als Antwort auf die latente endotheliale Schädigung und die hämodynamische Mikroangiopathie zu sein. Die überschießenden Reparaturmechanismen wurden in der Lunge ebenfalls bei vielen Formen interstitieller Lungenerkrankungen beschrieben. So zeigten wir die Bedeutung der intussuszeptiven Angiogenese insbesondere bei den morphologischen Subtypen der nichtspezifischen interstitiellen Pneumonie (NSIP) und der alveolären Fibroelastose (AFE) [22], aber auch in Formen der pulmonalen Hypertonie und bei pul- monalen venookklusiven Erkrankungen auf [24]. Obgleich die molekularen Mechanismen, die dem fibrotischen Umbau nach Mikrothrombembolien zugrunde liegen, noch unzureichend verstanden sind, legen die bisherigen Ergebnisse eine entscheidende Rolle der endothelialmesenchymalen Transition nahe, zumal die Lunge neben der Leber und dem Knochenmark ein Reservoir von endothelialen Progenitorzellen zu sein scheint [25]. Eine besondere Rolle wird residualen und zirkulierenden endothelialen Progenitorzellen in der Rekonvaleszenzphase von Patienten mit Heilungsverlauf nach akutem Lungenversagen oder bei bakterieller Pneumonie zugeschrieben $[26,27]$.

\section{Kardiovaskuläre Beteiligung}

Auch wenn bei COVID-19 die respiratorischen Einschränkungen akutmedizinisch die maßgebliche klinische Herausforderung darstellen, lassen sich bei zahlreichen COVID-19-Patienten im Laufe der Erkrankung auch kardiovaskuläre Komplikationen feststellen [24]. Klinisch wird diese kardiale Mitbeteiligung meist über eine akute Erhöhung der Troponinspiegel evident, welche bei ungefähr jedem zehnten hospitalisierten Patienten 
beobachtet wird und die wahrscheinlich in einem direkten Zusammenhang mit einer erhöhten Mortalität anzusehen ist [28]. Als Ursache für diese myokardiale Schädigung wird ein möglicher direkter toxischer Effekt über eine aberrante systemische Freisetzung von Chemokinen (wie z.B. TNF- $\alpha$, SDF1, CCL2, CCL3 oder CXCL10), Interferonen und Interleukinen (wie z.B. Interleukin 6) im Rahmen des sog. Zytokinsturmes diskutiert [29]. Auch wenn kardiovaskuläre Vorerkrankungen als Risikofaktor für einen schweren Verlauf der COVID-19Erkrankung angesehen werden, könnte auch eine direkte Schädigung des Myokards durch die SARS-CoV-2-Virämie ursächlich sein. Untermauert wird diese Hypothese von einer Studie an Autopsien, die im Herzmuskelgewebe eine direkte Replikation von SARS-CoV-2 nachweisen konnte. Erstaunlicherweise wurde in Studien aber überwiegend die Replikation des Virus in interstitiellen Zellen wie Makrophagen beobachtet. In einer weiteren Autopsiestudie wurde in über $85 \%$ der Fälle rechts- wie auch linksventrikulär eine diffuse Infiltration durch CD68+-positive Makrophagen beschrieben. Pathognomonisch ist die diffuse Makrophageninfiltration für COVID-19 (- Abb.5) aber nicht, da diese u.a. auch häufig, z. B. bei Verstorbenen, im Rahmen einer bakteriellen Sepsis gesehen wird und über die systemischen Spiegel von proinflammatorischen Zytokinen erklärt werden kann [28, 29]. In einem weitaus kleineren Anteil von Patienten wird eine äußerst seltene lymphozytäre Myokarditis beobachtet, welche multifokal eine Expression von CD3+-TLymphozyten aufweist und keine Riesenzellen, Granulome oder Eosinophilie beinhaltet. Fallstudien, die von einem fulminanten Verlauf einer Myokarditis oder von Takotsubo-Kardiomyopathien berichten, scheinen Einzelfälle zu sein, die nicht unbedingt in direkten kausalen Zusammenhang zu einer SARS-CoV-2Infektion stehen [28, 29]. Fulminante Myokarditisverläufe, wie sie nach anderen viralen Atemwegserkrankungen, wie z. B. Influenza-[30] oder ParvovirusB19-Infektionen, [21] gefunden werden, scheint es im Verlauf der COVID-19-
Erkrankung nicht in vergleichbarem Umfang zu geben.

\section{Fazit für die Praxis}

- COVID-19 und Influenza führen im Erkrankungsverlauf beide zu einem diffusen Alveolarschaden mit exsudativer Alveolitis und Organisationsphänomenen wie einer organisierenden Pneumonie (• Abb. 6)

- COVID-19 stellt eine vaskuläre Systemerkrankung dar und weist im Rahmen der Infektion der Alveolarepithelzellen ebenso eine prominente Gefäßbeteiligung im Sinne einer Endothelialitis mit systemischer thrombotischer Mikroangiopathie auf (• Abb. 6).

- Diese angiozentrische Entzündung induziert einen aberranten Reparaturmechanismus mit Gefäßneubildung mittels intussuszeptiver Angiogenese (- Abb. 6).

- COVID-19 führt in schwerwiegenden Verläufen auch zu kardiovaskulären Schädigungen, die in ihrem vollen Umfang noch unverstanden sind und meist durch eine diskrete Infiltration von Makrophagen und wenigen T-Lymphozyten charakterisiert sind.

\section{Korrespondenzadresse}

PD Dr. Maximilian Ackermann

Institut für Pathologie und Molekularpathologie, Helios Universitätsklinikum Wuppertal, Universität Witten-Herdecke

Heusnerstr. 40, 42283 Wuppertal, Deutschland maximilian.ackermann@helios-gesundheit.de

Prof. Dr. Danny D. Jonigk, FRCPath

Institut für Pathologie, Medizinische Hochschule Hannover (MHH)

Carl-Neuberg-Str. 1, 30625 Hannover, Deutschland

Jonigk.Danny@MH-Hannover.de

Förderung. Diese Arbeit wurde unterstützt durch das Deutsche Register für COVID-19 Obduktionen (DeRegCOVID, www.DeRegCOVID.ukaachen. de; finanziert durch das Bundesministerium für Gesundheit - ZMVI1-2520COR201) und das Bundesministerium für Bildung und Forschung im Rahmen des Netzwerkes Universitätsmedizin (DEFEAT PANDEMIcs, 01KX2021). Grants: PD Dr. Maximilian Ackermann: National Institutes of Health (HL94567 and HL134229); Prof. Dr. Danny Jonigk: European Research Council consolidation Grants (XHale; 771883, D3)
Einhaltung ethischer Richtlinien

Interessenkonflikt. M. Ackermann, C. Werlein, F. Länger, M. P. Kühnel und D. D. Jonigk geben an, dass kein Interessenkonflikt besteht.

Für diesen Beitrag wurden von den Autoren keine Studien an Menschen oder Tieren durchgeführt. Für die aufgeführten Studien gelten die jeweils dort angegebenen ethischen Richtlinien.

\section{Literatur}

1. Forum of International Respiratory Societies (2017) The global impact of respiratory disease, 2 . Aufl. European Respiratory Society, Sheffield

2. Boni MF, Lemey $P$, Jiang $X$, Lam TT, Perry BW, Castoe TA, Rambaut A, Robertson DL (2020) Evolutionary origins of the SARS-CoV-2 sarbecovirus lineage responsible for the COVID-19 pandemic. Nat Microbiol 5(11):1408-1417. https://doi.org/ 10.1038/s41564-020-0771-4

3. Ackermann M, Verleden SE, Kuehnel M, Haverich $A$ Welte T, Laenger F, Vanstapel A, Werlein C, Stark H, Tzankov A, Li WW, Li VW, Mentzer SJ, Jonigk D (2020) Pulmonary vascular endothelialitis, thrombosis, and angiogenesis in Covid-19. N Engl J Med 383(2):120-128. https://doi.org/10.1056/ NEJMoa2015432

4. Gupta A, Madhavan MV, Sehgal K, Nair N, Mahajan S, Sehrawat TS, Bikdeli B, Ahluwalia N, Ausiello JC, Wan EY, Freedberg DE, Kirtane AJ, Parikh SA, Maurer MS, Nordvig AS, Accili $D$, Bathon JM, Mohan S, Bauer KA, Leon MB, Krumholz HM, Uriel N, Mehra MR, Elkind MSV, Stone GW, Schwartz A, Ho DD, Bilezikian JP, Landry DW (2020) Extrapulmonary manifestations ofCOVID-19. Nat Med 26(7):1017-1032

5. Ortiz-Prado E, Simbaña-Rivera K, Gómez-Barreno L, Rubio-Neira M, Guaman LP, Kyriakidis NC, Muslin C, Jaramillo AMG, Barba-Ostria C, CevallosRobalino D, Sanches-SanMiguel H, Unigarro L, Zalakeviciute R, Gadian N, López-Cortés A (2020) Clinical, molecular, and epidemiological characterization of the SARS-CoV-2 virus and the Coronavirus Disease 2019 (COVID-19), a comprehensive literature review. Diagn Microbiol Infect Dis 98(1):115094. https://doi.org/10.1016/j. diagmicrobio.2020.115094

6. Lax SF, Skok K, Zechner P, Kessler HH, Kaufmann N, Koelblinger C, Vander K, Bargfrieder U, Trauner M (2020) Pulmonary arterial thrombosis in COVID19 with fatal outcome : results from a prospective, single-center, clinicopathologic case series. Ann Intern Med 173(5):350-361. https://doi.org/10. 7326/M20-2566

7. Kommoss FKF, Schwab C, Tavernar L, Schreck J, Wagner WL, Merle U, Jonigk D, Schirmacher $\mathrm{P}$ Longerich T (2020) The pathology of severe COVID-19-related lung damage. Dtsch Arztebl Int 117(29-30):500-506. https://doi.org/10.3238/ arztebl.2020.0500

8. Terpos E, Ntanasis-Stathopoulos I, Elalamy I, Kastritis E, Sergentanis TN, Politou M, Psaltopoulou T, Gerotziafas G, Dimopoulos MA (2020) Hematological findings and complications of COVID-19. Am J Hematol 95(7):834-847. https://doi.org/10.1002/ ajh.25829

9. Ackermann M, Mentzer SJ, Kolb M, Jonigk D (2020) Inflammation and intussusceptive angiogenesis in COVID-19: everything in and out of flow. 
Eur Respir J. https://doi.org/10.1183/13993003. 03147-2020

10. Khakpour S, Wilhelmsen K, Hellman J (2015) Vascular endothelial cell Toll-like receptor pathways in sepsis. Innate Immun 21(8):827-846. https://doi. org/10.1177/1753425915606525

11. Leppkes M, Knopf J, Naschberger E, Lindemann A, Singh J, Herrmann I, Stürzl M, Staats L, Mahajan A, Schauer C, Kremer AN, VölkI S, Amann K, Evert K, Falkeis C, Wehrfritz A, Rieker RJ, Hartmann A, Kremer AE, Neurath MF, Muñoz LE, Schett G, Herrmann M (2020) Vascular occlusion by neutrophil extracellular traps in COVID-19. EBioMedicine 58(2020):102925-102931. https:// doi.org/10.1016/j.ebiom.2020.102925

12. Libby P, Lüscher T (2020) COVID-19 is, in the end, an endothelial disease. Eur Heart J 41(32):3038-3044. https://doi.org/10.1093/eurheartj/ehaa623

13. Yan R, Zhang Y, Li Y, Xia L, Guo Y, Zhou Q (2020) Structural basis for the recognition of SARS-CoV-2 by full-length human ACE2. Science 367(6485):1444-1448. https://doi.org/10.1126/ science.abb2762

14. Hamming I, Timens W, Bulthuis ML, Lely AT, Navis G, van Goor H (2004) Tissue distribution of ACE2 protein, the functional receptor for SARS coronavirus. A first step in understanding SARS pathogenesis. J Pathol 203(2):631-637. https:// doi.org/10.1002/path. 1570

15. Cortese M, Lee JY, Cerikan B, Neufeldt CJ, Oorschot VMJ, Köhrer S, Hennies J, Schieber NL, Ronchi P, Mizzon G, Romero-Brey I, SantarellaMellwig R, Schorb M, Boermel M, Mocaer K, Beckwith MS, Templin RM, Gross V, Pape C, Tischer C, Frankish J, Horvat NK, Laketa V, Stanifer M, Boulant S, Ruggieri A, ChatelChaix L, Schwab Y, Bartenschlager R (2020) Integrative imaging reveals SARS-coV-2-induced reshaping of subcellular morphologies. Cell Host Microbe 28(6):853-866.e5. https://doi.org/10. 1016/j.chom.2020.11.003

16. Jin $Y$, Ji W, Yang $H$, Chen S, Zhang W, Duan $G$ (2020) Endothelial activation and dysfunction in COVID-19: from basic mechanisms to potential therapeutic approaches. Signal Transduct Target Ther 5(1):293. https://doi.org/10.1038/s41392020-00454-7

17. Ackermann M, Mentzer SJ, Jonigk D (2020) Pulmonary vascular pathology in Covid-19. Reply. N Engl J Med 383(9):888-889. https://doi.org/10. 1056/NEJMc2022068

18. Mentzer SJ, Konerding MA (2014) Intussusceptive angiogenesis: expansion and remodeling of microvascular networks. Angiogenesis 17(3):499-509. https://doi.org/10.1007/s10456-014-9428-3

19. Ackermann M, Houdek JP, Gibney BC, Ysasi A, Wagner W, Belle J, Schittny JC, Enzmann F, Tsuda A, Mentzer SJ, Konerding MA (2014) Sprouting and intussusceptive angiogenesis in postpneumonectomy lung growth: mechanisms of alveolar neovascularization. Angiogenesis 17(3):541-551. https://doi.org/10.1007/s10456013-9399-9

20. Ackermann M, Tsuda A, Secomb TW, Mentzer SJ, Konerding MA (2013) Intussusceptive remodeling of vascular branch angles in chemically-induced murine colitis. Microvasc Res 87:75-82. https://doi. org/10.1016/j.mvr.2013.02.002

21. Ackermann $M$, Wagner WL, Rellecke $P$, Akhyari $P$, Boeken U, Reinecke P (2020) Parvovirus B19induced angiogenesis in fulminant myocarditis. Eur Heart J 41(12):1309. https://doi.org/10.1093/ eurheartj/ehaa092
22. Ackermann M, Stark H, Neubert L, Schubert $S$, Borchert P, Linz F, Wagner WL, Stiller W, Wielpütz $M$, Hoefer A, Haverich A, Mentzer SJ, Shah HR, Welte T, Kuehnel M, Jonigk D (2020) Morphomolecular motifs of pulmonary neoangiogenesis in interstitial lung diseases. Eur Respir J 55(3):1900933-1900912. https://doi.org/ 10.1183/13993003.00933-2019

23. Ackermann M, Morse BA, Delventhal V, Carvajal IM Konerding MA (2012) Anti-VEGFR2 and antiIGF-1R-Adnectins inhibit Ewing's sarcoma A673xenograft growth and normalize tumor vascular architecture. Angiogenesis 15(4):685-695. https:// doi.org/10.1007/s10456-012-9294-9

24. Neubert L, Borchert $P$, Shin HO, Linz F, Wagner WL, Warnecke $G$, Laenger $F$, Haverich $A$, Stark $H$, Hoeper MM, Kuehnel M, Ackermann M, Jonigk D (2019) Comprehensive three-dimensional morphology of neoangiogenesis in pulmonary venoocclusive disease and pulmonary capillary hemangiomatosis. JPathol Clin Res 5(2):108-114. https:// doi.org/10.1002/cjp2.125 (Epub 2019Feb 27)

25. Schniedermann J, Rennecke M, Buttler K, Richter G, Städtler AM, Norgall S, Badar M, Barleon B, May T, Wilting J, Weich HA (2010) Mouse lung contains endothelial progenitors with high capacity to form blood and lymphatic vessels. BMC Cell Biol 11:50. https://doi.org/10.1186/1471-2121-11-50

26. Rafat N, Tönshoff B, Bierhaus A, Beck GC (2013) Endothelial progenitor cells in regeneration after acute lung injury: do they play a role? Am J Respi Cell Mol Biol 48(4):399-405. https://doi.org/10. 1165/rcmb.2011-0132TR

27. Yamada M, Kubo H, Ishizawa K, Kobayashi S, Shinkawa M, Sasaki H (2005) Increased circulating endothelial progenitor cells in patients with bacterial pneumonia: evidence that bone marrow derived cells contribute to lung repair. Thorax 60(5):410-413. https://doi.org/10.1136/thx.2004. 034058

28. Lindner $D$, Fitzek $A$, Bräuninger $H$, Aleshcheva $G$ Edler C, Meissner K, Scherschel K, Kirchhof $P$, Escher F, Schultheiss HP, Blankenberg S, Püschel K Westermann D (2020) Association of cardiac infection with SARS-coV-2 in confirmed COVID-19 autopsy cases. JAMA Cardiol 27:e203551. https:// doi.org/10.1001/jamacardio.2020.3551

29. Basso C, Leone O, Rizzo S, De Gaspari M, van der Wal AC, Aubry MC, Bois MC, Lin PT, Maleszewski JJ, Stone JR (2020) Pathological features of COVID-19-associated myocardial injury: a multicentre cardiovascular pathology study. Eur Heart J 41(39):3827-3835. https://doi. org/10.1093/eurheartj/ehaa664

30. Mamas MA, Fraser D, Neyses L (2008) Cardiovascular manifestations associated with influenza virus infection. Int J Cardiol 130(3):304-309. https://doi. org/10.1016/j.ijcard.2008.04.044

\section{Immuntherapie in der Uroonkologie}

Retz, M., Gschwend, J. E.

1.Auflage, 365 Seiten, inkl. Ebook, 79,99 EUR, ISBN 978-3-662-60977-4

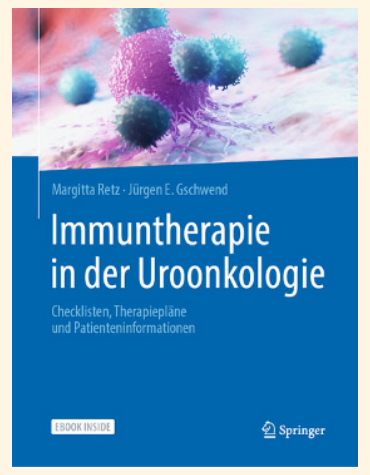

"Immuntherapie in der Uroonkologie" vereinfacht dem Arzt die onkologische Therapie durch klare Handlungsanweisungen in Form von Checklisten, Patienteninformationsblätter und Therapieplänen

In einzigartiger Form bündelt das Buch das aktuelle Wissen, welches der Arzt für die sichere Behandlung zur Versorgung von urologischen Tumorpatienten benötigt. Zahlreiche Checklisten, Tabellen, Therapiepläne und Patienteninformationen machen ein schnelles Nachschlagen möglich. Erstmalig wurde das Nebenwirkungsmanagement von immunvermittelten Toxizitäten auf der Grundlage von internationalen Leitlinien in Tabellenform aufgelistet. Weitere Schwerpunkte sind die Pharmakologie von Immun- und Targettherapeutika unter besonderer Berücksichtigung der Nebenwirkungsprofile und Kontraindikationen. Die übersichtlich gestalteten Therapieschemata und Patienteninformationen können als Vorlage für die eigene Arbeit genutzt werden - sie sind druckbar und kopierbar. Für den direkten Einsatz während der Therapie erhalten Sie zusätzlich das eBook. 